\title{
Deficiency of gap junction composed of connexin 43 contributes to oxaliplatin resistance in colon cancer cells
}

\author{
MIN SU and QI ZHANG \\ Department of Oncology, Hospital Affiliated to Hubei University of Arts and \\ Science/Xiangyang Central Hospital, Xiangyang, Hubei 441021, P.R. China
}

Received April 7, 2016; Accepted January 26, 2017

DOI: $10.3892 / 01.2017 .6598$

\begin{abstract}
Although comprehensive strategies in the treatment of colorectal cancer have been developed for a number of years, the five-year survival rate of metastatic colon cancer remains less than $10 \%$. Oxaliplatin, a commonly used chemotherapeutic agent for metastatic colon cancer, improves the response rate of patients and prolongs patients' progression-free survival. However, the generation of resistance limits the clinical application of oxaliplatin, and the mechanisms of this remain unclear. The present study mainly investigated the effect of the gap junction (GJ) composed of connexin43 (Cx43) on oxaliplatin cytotoxicity in colon cancer cells. Three different methods with distinct mechanisms were used to change the function of Cx43 GJs, including cell culture at different densities, pretreatment with a specific inhibitor or enhancer, and special gene knockdown, to observe the cytotoxicity of oxaliplatin and the level of reactive oxygen species (ROS) mediated by Cx43 GJs. The results revealed that the cytotoxicity of oxaliplatin and the level of ROS were decreased with the downregulation of $\mathrm{Cx} 43$ GJ function, but exacerbated with the upregulation of Cx 43 GJ function. Moreover, ROS scavenging with N-acetyl-L-cysteine and apocynin decreased the cytotoxicity of oxaliplatin. We concluded that the loss of GJ composed of $\mathrm{Cx} 43$ contributed to the resistance of oxaliplatin in colon cancer cells, and the mechanism was associated with intracellular ROS alternation.
\end{abstract}

\section{Introduction}

Colorectal cancer (CRC) is considered to be one of the most frequent causes of cancer-related morbidity and mortality worldwide $(1,2)$. Although comprehensive strategies in the treatment of CRC have been developed for a number of

Correspondence to: DrQiZhang, Department of Oncology, Hospital Affiliated to Hubei University of Arts and Science/Xiangyang Central Hospital, 136 Jingzhou Street, Xiangcheng, Xiangyang, Hubei 441021, P.R. China

E-mail: 360491866@qq.com

Key words: colorectal cancer, connexin43, oxaliplatin, resistance, reactive oxygen species years, the five-year survival rate of metastatic colon cancer is only $10 \%$ (3). In the clinical treatment of metastatic colon cancer, oxaliplatin is commonly used as an essential chemotherapeutic agent, and not only improves the response rate of patients, but also prolongs patients' progression-free survival. Nevertheless, more than $40 \%$ of patients still develop significant resistance $(3,4)$. Therefore, how to overcome resistance to oxaliplatin is a key scientific problem to be solved in the treatment of metastatic colon cancers.

Connexins are integral membrane proteins, six of which make up a hemi-channel. Two hemi-channels in neighboring cells dock together to form an integral gap junction (GJ). The GJ enables cells to exchange ions and small molecules (with a molecule weight less than $1 \mathrm{kDa}$ ) directly, including calcium, glutathione, cyclic adenosine monophosphate and cyclic guanosine monophosphate. Molecules transferred through the GJ are essential for numerous physiological and pathological events $(5,6)$. The connexin gene family constitutes 21 isoforms, the most significant of which is $\mathrm{Cx} 43$ (6). The loss of Cx43 is extremely common in the development of cancers and is even involved in advanced stages of tumor progression. The deficiency of $\mathrm{Cx} 43$ also contributes to the resistance of chemotherapeutic agents (7). It has been reported that decreased levels of $\mathrm{Cx} 43 \mathrm{GJ}$ are associated with the resistance of temozolomide and cisplatin targeting glioblastoma or lung adenocarcinoma $(8,9)$. Furthermore, Sirnes et al indicated that $\mathrm{Cx} 43$ may be a CRC tumor suppressor protein that predicts clinical outcome (7). Thus, we supposed that the resistance of oxaliplatin to metastatic colon cancers might be involved in the alternation of $\mathrm{Cx} 43 \mathrm{GJ}$ function, and explored the mechanisms involved in this.

Reactive oxygen species are one of the significant elements that may be transferred between neighboring cells through the GJ (5). More significantly, Lin et al observed that ROS-related mechanisms increased oxaliplatin sensitivity in human colorectal cancers (10). Thus, in the present study, we present the first evidence that ROS distribution mediated by $\mathrm{Cx} 43$ contributes to the resistance of oxaliplatin in the treatment of human CRC.

\section{Materials and methods}

Cell line and cell culture. The human colon cancer cell line COLO320 was obtained from the American Type Culture 
Collection (Manassas, VA, USA) and cultured in RPMI-1640 medium (Invitrogen Life Technologies, Carlsbad, CA, USA) supplemented with 10\% fetal bovine serum (Invitrogen Life Technologies) and $100 \mathrm{U} / \mathrm{ml}$ penicillin-streptomycin (Invitrogen Life Technologies) in a $5 \% \mathrm{CO}_{2}$ incubator with $90 \%$ humidity at $37^{\circ} \mathrm{C}$ (Thermo Fisher Scientific, Waltham, MA, USA).

Colony-forming assay. Colony-forming assay was used to detect cytotoxicity dependent on GJ. At high cell density culture, cells were seeded at 100,000 cells $/ \mathrm{cm}^{2}$. When cells were exposed to drugs, the cultures were 70 to $100 \%$ confluent and GJs were formed. At low cell density culture, cells were seeded at 10,000 cells $/ \mathrm{cm}^{2}$. When cells were exposed to drugs, GJs were not formed. Cells were treated with oxaliplatin (0-125 $\mu \mathrm{M}$, Sigma-Aldrich, St. Louis, MO, USA) for $24 \mathrm{~h}$, and then washed with RPMI-1640 medium. Next, cells were harvested by trypsinization (Invitrogen Life Technologies), counted, diluted and seeded into six-well dishes at 100 cells $/ \mathrm{cm}^{2}$. After 7 days, cells were stained with crystal violet (Sigma-Aldrich) and assessed. Colonies containing more than 50 cells were scored (11).

Chemical treatment, lactate dehydrogenase ( $L D H)$ and ROS assay. COLO320 cells were pretreated with connexin mimetic peptide Gap26 at $300 \mu \mathrm{M}$ for $1 \mathrm{~h}$ (Sigma-Aldrich) to inhibit $\mathrm{Cx} 43$ channel function, as well as retinoic acid (RA) at $10 \mu \mathrm{M}$ for $24 \mathrm{~h}$ (Sigma-Aldrich) to enhance $\mathrm{Cx} 43$ channel function prior to the different assays. $\mathrm{N}$-acetyl-L-cysteine (NAC; $5 \mathrm{mM}, 24 \mathrm{~h}$; Sigma-Aldrich) and apocynin (AP; $10 \mu \mathrm{M}, 24 \mathrm{~h}$; Sigma-Aldrich) were used to scavenge ROS. The corresponding solvent of these chemicals was dimethyl sulfoxide (DMSO; Sigma-Aldrich). Cell growth was determined via a colony-forming assay. LDH assays (Roche Diagnostics, Indianapolis, IN, USA) were carried out according to the manufacturer's instructions. Intracellular ROS production was estimated using 2,7-dichlorofluorescein diacetate (Sigma-Aldrich).

Cx43 knockdown with small interfering RNA (siRNA) transfection. Two specific siRNAs (GCTGGTTACTGGTGA CAGA and CCGCAATTACAACAAGCAA; siRNA1-Cx43 and siRNA2-Cx43) targeting the $\mathrm{Cx} 43$ gene were designed to decrease $\mathrm{Cx} 43$ expression. Nonspecific siRNA-Cx43 was used as a negative control (NC). The siRNA and NC were purchased from Guangzhou RiboBio Co., Ltd. (Guangzhou, China). Lipofectamine 2000 (Invitrogen Life Technologies) was used to transfect siRNA and NC into the cells according to the manufacturer's instructions (12).

'Parachute' dye-coupling assay. The 'parachute' dye-coupling method was used to detect the GJ function. Donor cells were labeled with $5 \mu \mathrm{M}$ CM-DiI (Invitrogen Life Technologies) and $5 \mu \mathrm{M}$ calcein-acetoxymethyl ester (Invitrogen Life Technologies). CM-DiI did not spread to coupled cells, but calcein-acetoxymethyl ester was able to transform into the neighboring cells through the GJ. Donor cells were seeded onto the receiver cells at a 1:150 donor/receiver ratio to form the GJ. Four h later, GJ function was examined with a fluorescence microscope (Eclipse E800, Nikon Corporation, Tokyo,
Japan). The average number of receiver cells containing dye per donor cell was counted and normalized to that of control cultures (13).

Western blotting. Cells were washed three times with wash buffer $[0.01 \mathrm{~mol} / 1$ phosphate-buffered saline, $0.138 \mathrm{~mol} / 1$ $\mathrm{NaCl}, 0.02 \% \mathrm{NaN}_{3}$ (pH 7.4)] and then incubated with lysis buffer for 2 h (Nanjing Keygen Biotech Co., Ltd., Nanjing, China) at $4^{\circ} \mathrm{C}$ using $0.05 \mathrm{ml} / \mathrm{cm}^{2}$. The bicinchoninic acid method (Nanjing Keygen Biotech Co., Ltd.) was used to measure protein concentrations. Cell lysates $(30 \mu \mathrm{g})$ were separated by SDS-PAGE on 10\% Tris-glycine mini-gels (Invitrogen Life Technologies) and transferred onto polyvinylidene difluoride membrane (Bio-Rad Laboratories, Inc., Hercules, CA, USA). We used 5\% non-fat dry milk (Sigma-Aldrich) to block the membranes at room temperature for $30 \mathrm{~min}$. After that, the membranes were immunoblotted using anti-Cx43 antibody (1:4,000; mouse monoclonal $\mathrm{Cx} 43$ antibody raised against humans; cat. no. C8093; Sigma-Aldrich) overnight at $4^{\circ} \mathrm{C}$. After several washes, the membranes were incubated for $1 \mathrm{~h}$ at room temperature with anti-mouse horseradish peroxidase (HRP)-conjugated secondary antibodies (1:4,000; goat polyclonal antibody raised against mouse $\mathrm{IgG}$; cat. no. M6898; Sigma-Aldrich). Mouse monoclonal anti- $\beta$-actin antibody raised against humans (cat. no. A1978; Sigma-Aldrich) was immunoblotted $(1: 10,000)$ at $4^{\circ} \mathrm{C}$ overnight. Anti-mouse HRP-conjugated secondary antibodies (goat polyclonal antibody raised against mouse IgG; cat. no. M6898; Sigma-Aldrich) was used at 1:10,000 for $1 \mathrm{~h}$ at room temperature. All protein bands were detected with an enhanced chemiluminescence system (KGP1125; Nanjing KeyGen Biotech. Co., Ltd.) and estimated using Alpha View software (version 2.2.14407; ProteinSimple, Santa Clara, CA, USA).

Statistical analysis. Statistical analysis was performed using SPSS 15.0 software (SPSS, Inc., Chicago, IL, USA). Multiple comparisons among groups were analyzed using one-way analysis of variance, followed by Tukey post hoc comparisons. $\mathrm{P}<0.05$ was considered to indicate a statistically significant difference.

\section{Results}

Effects of oxaliplatin on cell survival, $L D H$ release and intracellular ROS are all dependent on cell density. In order to explore the effects of GJ function on oxaliplatin cytotoxicity, COLO320, a type of human colon cancer cell line, was cultured at low and high density. At low-density cell culture, the cells were not in contact with each other and no GJs were formed when exposed to different chemicals. However, at high-density cell culture, cells were confluent, which allowed significant formation of GJs. This method was therefore used to explore the function of the GJ. Fig. 1A and B reveal that when cells were in contact with one another at high-density cell culture, oxaliplatin cytotoxicity was substantially greater than at low-density cell culture. This manifested as lower clonogenic survival and a greater extent of LDH release in the high-density condition than in the low-density condition. These results indicated that oxaliplatin cytotoxicity was density-dependent, being more significant at high-density 

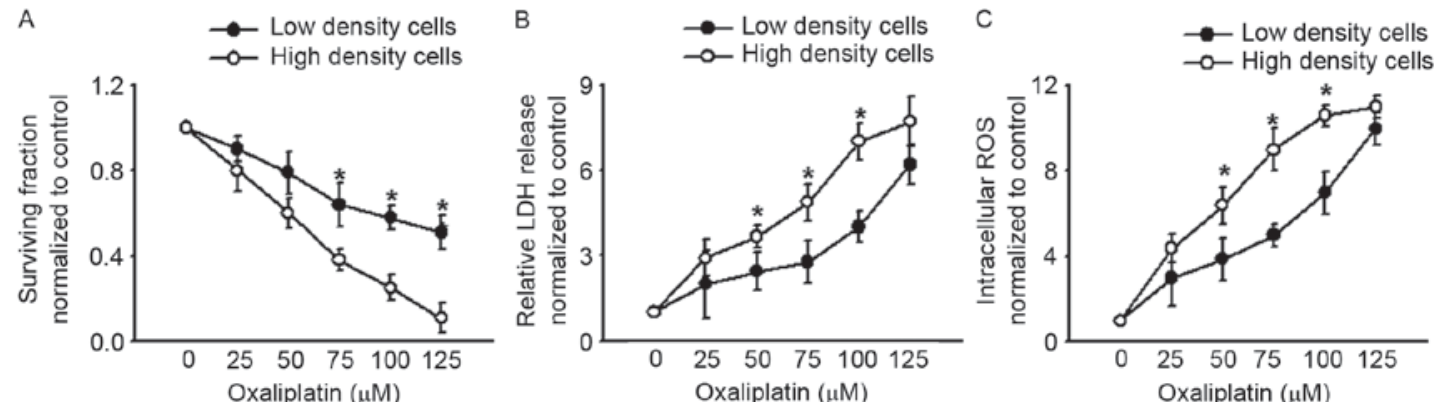

Figure 1. COLO320 cell clonogenic survival, LDH release and intracellular ROS are cell density-dependent when exposed to oxaliplatin (0-125 $\mu \mathrm{M}, 24 \mathrm{~h})$ (A) COLO320 cell clonogenic survival at low and high cell density. (B) LDH release of COLO320 cells at low and high cell density. (C) Intracellular ROS of COLO320 cells at low and high cell density. $\mathrm{n}=4-7$; ${ }^{*} \mathrm{P}<0.05$, compared with high-density cell group. $\mathrm{LDH}$, lactate dehydrogenase; ROS, reactive oxygen species.
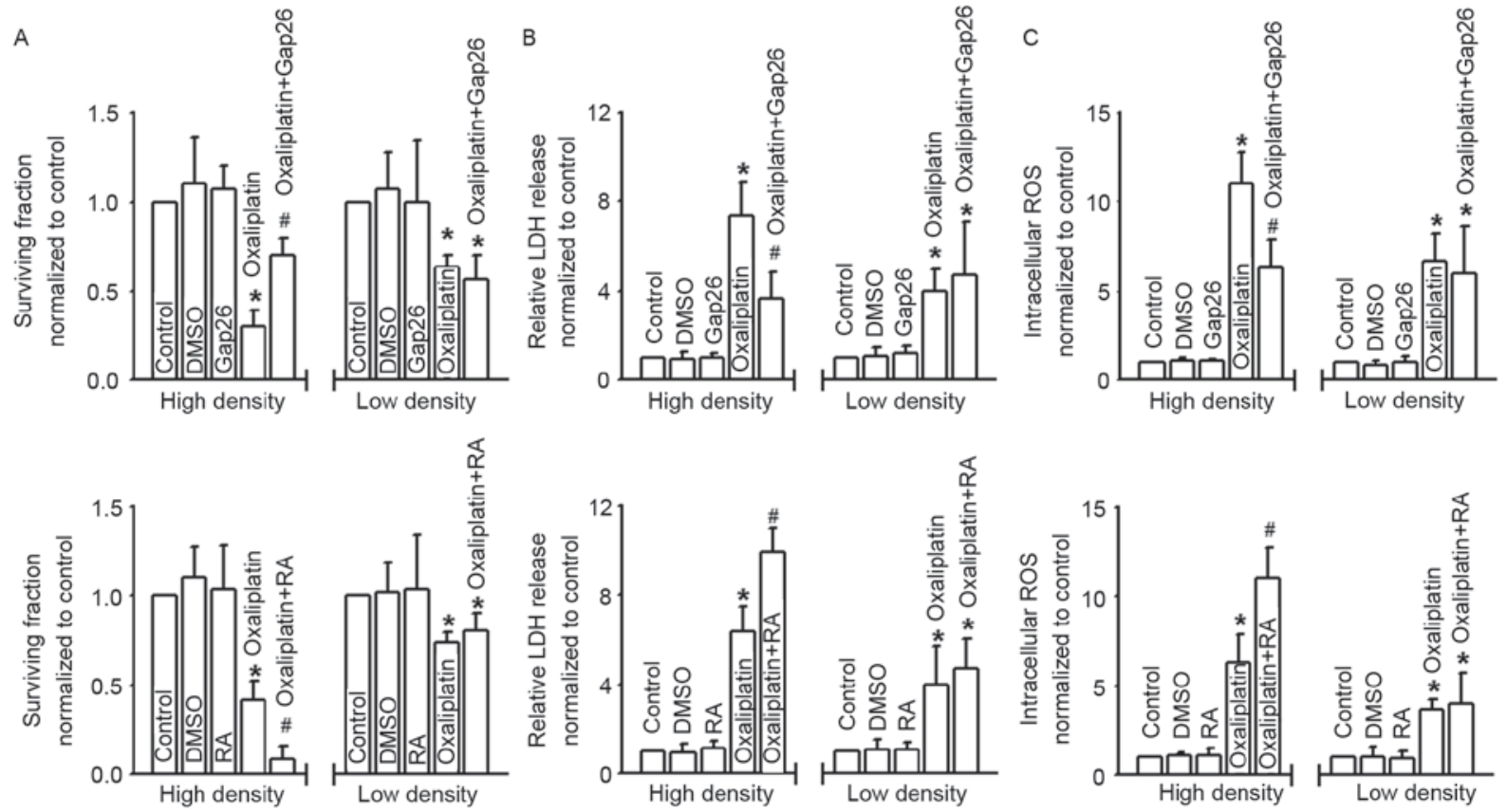

Figure 2. Gap junctions composed of $\mathrm{Cx} 43$ regulate the cytotoxicity of oxaliplatin. (A-C) Clonogenic survival, LDH release and intracellular ROS of COLO320 cells exposed to oxaliplatin $(100 \mu \mathrm{M}, 24 \mathrm{~h})$, at low or high density cell culture and co-incubated with or without Gap26 and RA. n=4-6; "P<0.05, compared with control group; ${ }^{~} \mathrm{P}<0.05$, compared with oxaliplatin group at high-density cell culture. Cx43, connexin43; LDH, lactate dehydrogenase; ROS, reactive oxygen species; DMSO, dimethyl sulfoxide; RA, retinoic acid.

cell culture where GJs were formed. As previously reported, density-dependent cytotoxicity was always relative with 'death signals' transferred between the neighboring cells via GJs: The most important 'death signals' was ROS (5). Thus, we determined the intercellular ROS of COLO320 at low- and high-density cell cultures when exposed to oxaliplatin. Fig. 1C reveals that intercellular ROS were increased more notably at high-density cell culture than at low-density cell culture, which suggests that ROS transfer mediated by GJ may play a significant part in oxaliplatin cytotoxicity.

GJs affect cell survival, $L D H$ release and intracellular ROS of COLO320 cells exposed to oxaliplatin. Density-dependent cell cytotoxicity was always regulated by the GJs, and $\mathrm{Cx} 43$ contributed to the cytotoxicity of antitumor drugs. In order to explore the effects of $\mathrm{Cx} 43 \mathrm{GJ}$ on oxaliplatin cytotoxicity targeting colon cancer cells, we used different methods with distinct mechanisms to manipulate the function of GJs composed of $\mathrm{Cx} 43$. Fig. 2A shows that the survival fraction of COLO320 cells detected with colony-forming assay was decreased in different degrees when subjected to oxaliplatin; however, at high-density cell culture (where GJs formed), the cells were much more sensitive to this commonly used chemotherapy drug. Moreover, the cytotoxicity of oxaliplatin was attenuated subsequent to pretreatment with Gap26, a specific inhibitor of $\mathrm{Cx} 43 \mathrm{GJ}$, but exacerbated following RA treatment. In contrast, at low-density cell culture (where GJs were not formed), the cytotoxicity of oxaliplatin demonstrated no significant difference when COLO320 cells were pretreated with Gap26 or RA. When cells were pretreated with oxaliplatin, LDH release and intracellular ROS of COLO320 cells increased significantly. At high-density cell culture, the levels of LDH release and intracellular ROS fluctuated as Cx43 GJ function changed, and this was modulated 

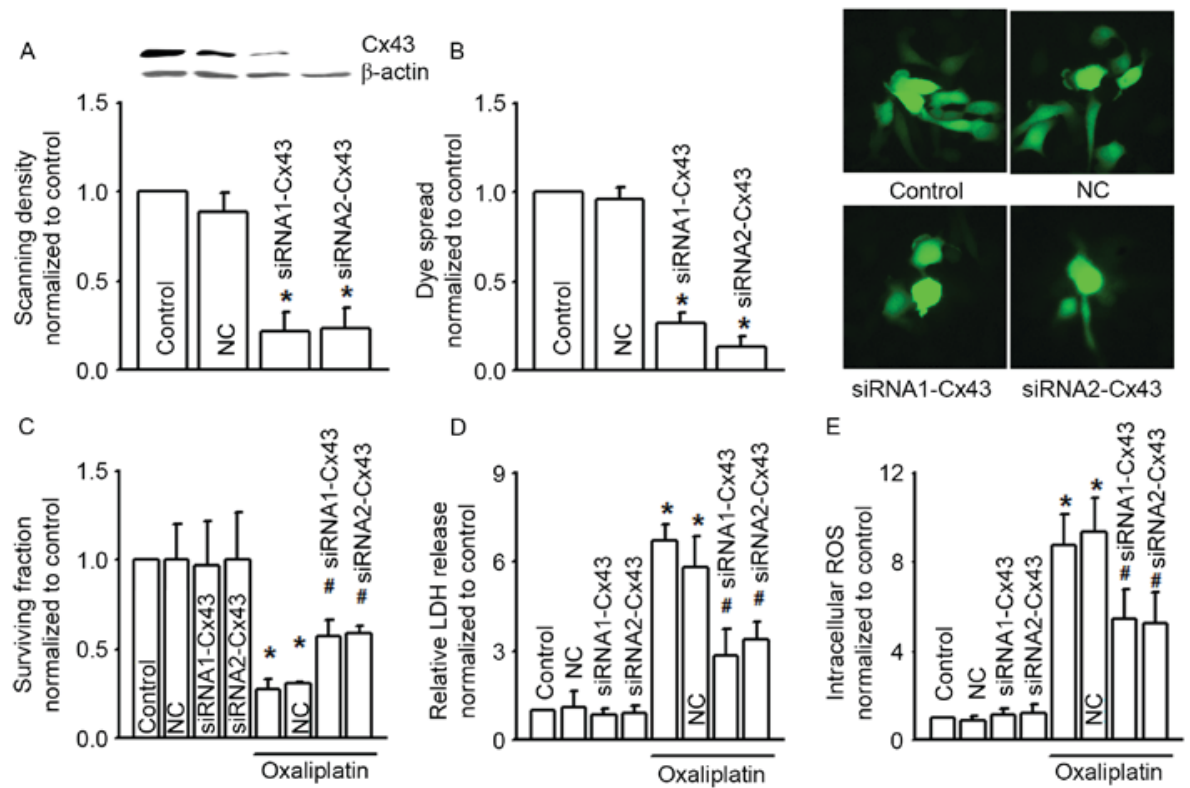

Figure 3. siRNA alters clonogenic survival, LDH release and intracellular ROS of COLO320 cells exposed to oxaliplatin (100 $\mu \mathrm{M}, 24 \mathrm{~h})$. (A) Two different specific siRNAs (siRNA1-Cx43, siRNA2-Cx43) decreased Cx43 expression. $n=3$; ${ }^{*} \mathrm{P}<0.05$, compared with NC group. (B) The two different specific siRNAs attenuated dye coupling of COLO320 cells. $n=3$; ${ }^{2} \mathrm{P}<0.05$, compared with NC group. (C-E) Clonogenic survival, LDH release and intracellular ROS of COLO320 cells exposed to oxaliplatin $(100 \mu \mathrm{M}, 24 \mathrm{~h})$, when pretreated with the two specific siRNAs. $\mathrm{n}=4-6$; ${ }^{*} \mathrm{P}<0.05$, compared with control group; ${ }^{*} \mathrm{P}<0.05$, compared with NC group. siRNA, small interfering RNA; LDH, lactate dehydrogenase; ROS, reactive oxygen species; Cx43, connexin43; NC, negative control.
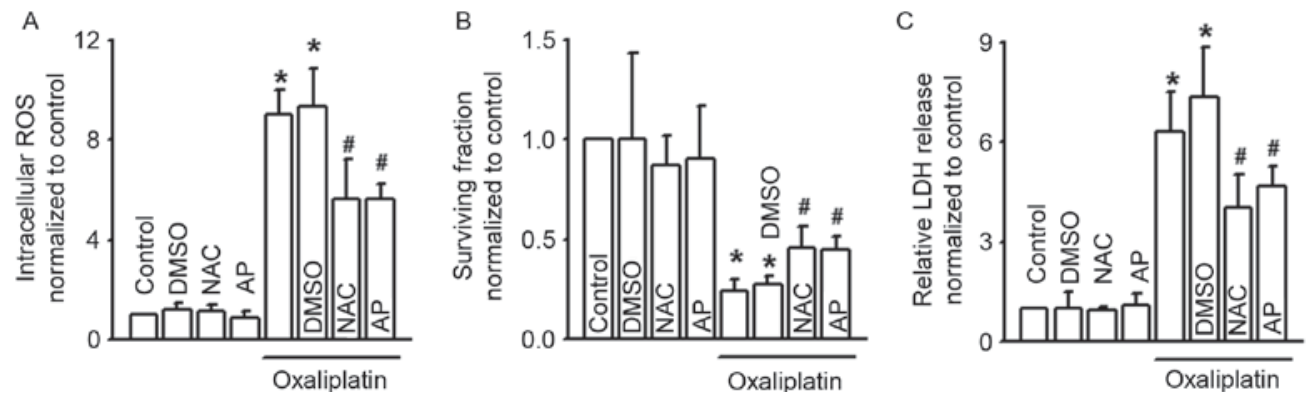

Figure 4. NAC and AP alter the clonogenic survival, LDH release and intracellular ROS of COLO320 cells exposed to oxaliplatin. (A-C) Intracellular ROS, clonogenic survival and LDH release of COLO320 cells exposed to oxaliplatin $(100 \mu \mathrm{M}, 24 \mathrm{~h})$ when pretreated with NAC (5 mM, $24 \mathrm{~h})$ and AP (10 $\mu \mathrm{M}$, 24 h). $\mathrm{n}=4-6$; ${ }^{*} \mathrm{P}<0.05$, compared with control group; " $\mathrm{P}<0.05$, compared with oxaliplatin group. NAC, N-acetyl-L-cysteine; AP, apocynin; LDH, lactate dehydrogenase; ROS, reactive oxygen species; DMSO, dimethyl sulfoxide.

by Gap26 and RA. However, at low-density cell culture, the change in Cx43 GJ function had no influence on the levels of LDH release and intracellular ROS. DMSO, the vehicle control of Gap26 and RA, had no effect on the parameters (Fig. 2B and C).

Cx43 gene knockdown attenuates COLO320 cell cytotoxicity of oxaliplatin. Figs. 1 and 2 illustrate that alternation of Cx43 GJ function affected the cytotoxicity of oxaliplatin in COLO320 cells. In order to confirm this result, we designed two different $\mathrm{Cx} 43$ siRNAs (siRNA1-Cx43 and siRNA2-Cx43) to knock down the expression of $\mathrm{Cx} 43$ specifically (Fig. 3A). As the expression of $\mathrm{Cx} 43$ was decreased, $\mathrm{Cx} 43 \mathrm{GJ}$ function was also notably decreased (Fig. 3B). Cx43 knockdown did not influence COLO320 survival fraction, LDH release and intracellular ROS per se, but Cx43 knockdown attenuated the cytotoxicity induced by oxaliplatin on COLO320, and increased the levels of oxaliplatin-induced LDH release and intracellular ROS, all of which indicated that Cx43 GJ function played a significant part in the cytotoxicity of oxaliplatin (Fig. 3C to E).

The antioxidants NAC and AP attenuate COLO320 cell cytotoxicity of oxaliplatin. It has been reported that the level of intracellular ROS affects the cytotoxicity of oxaliplatin (10). Our results (Figs. 1C, 2C and 3E) also revealed that Cx43 GJ function alternation changed the level of oxaliplatin-induced intracellular ROS. Thus, we supposed that modulation of the level of intracellular ROS by $\mathrm{Cx} 43 \mathrm{GJ}$ might contribute to the cytotoxicity of oxaliplatin. Fig. 4A demonstrates that two antioxidants, NAC and AP, effectively attenuated intracellular ROS increase induced by oxaliplatin. Moreover, pretreatment of NAC and AP increased the survival fraction of COLO320 cells, but decreased LDH release when exposed to oxaliplatin. Both of the above indicate that inhibition of intracellular ROS decreased the cytotoxicity of oxaliplatin (Fig. 4B and C). 


\section{Discussion}

The present study investigated the effect of Cx43 GJ on the cytotoxicity of oxaliplatin in colon cancer cells, as well as the mechanisms of its involvement in intracellular ROS alternation. We demonstrated that oxaliplatin worked in a cell density-dependent manner. At high-density cell culture (where GJs were formed), the cytotoxicity of oxaliplatin was attenuated subsequent to a decrease in Cx43 GJ function with the specific inhibitor of Cx43 GJ, Gap26, but exacerbated as Cx43 GJ function increased with the enhancer, RA. However, Gap26 and RA had no effect at low-density cell culture (where no GJs were formed). In conclusion, Cx43 plays a significant role in the cytotoxicity of oxaliplatin in COLO320, a colon cancer cell line. Significantly, we noted that intracellular ROS induced by oxaliplatin was also modulated by $\mathrm{Cx} 43$ GJs: when Cx43 GJ function was enhanced, intracellular ROS induced by oxaliplatin were increased; in contrast, intracellular ROS were decreased as Cx43 GJ function was attenuated. These results indicated that intracellular ROS alternation modulated by Cx43 GJs might contribute to the cytotoxicity of oxaliplatin in colon cancer cells.

CRC remains one of the leading causes of cancer-related mortality worldwide (14). Although therapeutic strategies have been improved in recent years, the five-year survival rate remains less than $10 \%$ for metastatic colon cancer (3). Oxaliplatin is one of the most commonly used chemotherapy drugs in colon cancer therapies. It is a third-generation platinum-based anti-neoplastic agent commonly used in the treatment of CRC $(15,16)$. Oxaliplatin application in the clinic has brought about notable improvements in the response rate and progression-free survival in advanced colon cancer, but $\sim 40 \%$ of patients still develop resistance $(3,17)$. Resistance generation limits oxaliplatin application in the course of clinical treatment. Thus, it is of vital clinical significance to investigate the resistance of chemotherapy drugs.

It has been reported that loss of GJs in the development of cancers contributes to the generation of resistance to chemotherapy drugs (18-20). Enhancement of GJ function increases the cytotoxicity of chemotherapy drugs (21). Conversely, inhibition of GJ function decreases the cytotoxicity of chemotherapy drugs $(22,23)$. However, the mechanisms of these processes remain unclear. GJ mediates the direct transfer of small molecules or electrical charge between neighboring cells, generating various effects on the different physiological and pathological issues processes. The most significant of the transferred molecular signals is called the 'death signal', which results in the amplification of cytotoxicity or apoptosis $(11,24)$. Chemotherapy drugs attack the cancer cells and result in cell death directly, and the attacked cells generate various types of toxic products, called death signals. The GJ is the main channel of death signals transferred between the neighboring cells. Death signals not only attack the neighboring cells directly, but also activate various signal pathways, resulting indirectly in cytotoxicity or apoptosis $(11,25)$. In summary, the effects of chemotherapy drugs are amplified by the transfer of death signals between the neighboring cells through the GJ, which is called the 'bystander effect' $(26,27)$. The present study also supports this hypothesis, as the cytotoxicity of oxaliplatin was attenuated subsequent to $\mathrm{CX} 43 \mathrm{GJ}$ function decrease with the specific inhibitor of Cx43 GJ, Gap26, but exacerbated as Cx43 GJ function increased with the enhancer, RA.

Thus far, the intrinsic quality of death signals has not been identified. Although the possibility of the involvement of calcium or other cell metabolites has been discussed for a number of years, direct proof is still lacking. We observed that ROS not only enhanced oxaliplatin sensitivity to CRC, but also contributed to epithelial-mesenchymal transition and cancer metastasis, which indicated that ROS may play an essential role in the development of cancers (10). Luo et al reported that GJs composed of Cx32 regulated the distribution of ROS (5). Therefore, we considered that ROS distribution modulated by GJs might contribute to the cytotoxicity of oxaliplatin. This hypothesis was supported by our finding that GJ function inhibition decreased the level of ROS and the cytotoxicity of oxaliplatin. Our results also indicated that ROS may be significant death signals.

As reported in previous studies, downregulation of $\mathrm{Cx} 43$ by siRNA resulted in a more aggressive growth of breast cancer cells, and Cx43 knockout mice were susceptible to chemicals inducing lung adenomas $(22,23)$. In contrast, enhancement of Cx43 expression promoted cancer cell restoration and differentiation in various types of tissue (27). Hence, it may be noted that $\mathrm{Cx} 43$ is one of the most significant tumor suppressors, and is associated with malignant behaviors of cancers. Our study demonstrates for the first time that GJs composed of Cx43 affect the cytotoxicity of oxaliplatin by modulating the levels of ROS in colon cancer cells. Moreover, Cx43 GJ function enhancement amplified the oxaliplatin cytotoxicity of colon cancer cells. This not only explains the possible mechanisms of resistance generation from another aspect, but also provides a potential target in colon cancer prevention and therapy.

\section{References}

1. Hirschi B, Gallmeier E, Ziesch A, Marschall M and Kolligs FT: Genetic targeting of B-RafV600E affects survival and proliferation and identifies selective agents against BRAF-mutant colorectal cancer cells. Mol Cancer 13: 122, 2014.

2. Arnold M, Sierra MS, Laversanne M, Soerjomataram I, Jemal A and Bray F: Global patterns and trends in colorectal cancer incidence and mortality. Gut 66: 686-691, 2017.

3. Tan S, Peng X, Peng W, Zhao Y and Wei Y: Enhancement of oxaliplatin-induced cell apoptosis and tumor suppression by 3-methyladenine in colon cancer. Oncol Lett 9: 2056-2062, 2015.

4. Fan F, Gray MJ, Dallas NA, Yang AD, Van Buren G II, Camp ER and Ellis LM: Effect of chemotherapeutic stress on induction of vascular endothelial growth factor family members and receptors in human colorectal cancer cells. Mol Cancer Ther 7: 3064-3070, 2008.

5. Luo C, Yuan D, Li X, Yao W, Luo G, Chi X, Li H, Irwin MG Xia Z and Hei Z: Propofol attenuated acute kidney injury after orthotopic liver transplantation via inhibiting gap junction composed of connexin 32. Anesthesiology 122: 72-86, 2015.

6. Chen X, Kong X, Zhuang W, Teng B, Yu X, Hua S, Wang S, Liang F, Ma D, Zhang S, et al: Dynamic changes in protein interaction between AKAP95 and Cx43 during cell cycle progression of A549 cells. Sci Rep 6: 21224, 2016.

7. Sirnes S, Bruun J, Kolberg M, Kjenseth A, Lind GE, Svindland A, Brech A, Nesbakken A, Lothe RA, Leithe E and Rivedal E: Connexin 43 acts as a colorectal cancer tumor suppressor and predicts disease outcome. Int J Cancer 131: 570-581, 2012.

8. Gielen PR, Aftab Q, Ma N, Chen VC, Hong X, Lozinsky S, Naus CC and Sin WC: Connexin 43 confers Temozolomide resistance in human glioma cells by modulating the mitochondrial apoptosis pathway. Neuropharmacology 75: 539-548, 2013.

9. Yu M, Zhang C, Li L, Dong S, Zhang N and Tong X: Cx43 reverses the resistance of A549 lung adenocarcinoma cells to cisplatin by inhibiting EMT. Oncol Rep 31: 2751-2758, 2014. 
10. Lin S, Lei K, Du W, Yang L, Shi H, Gao Y, Yin P, Liang X and Liu J: Enhancement of oxaliplatin sensitivity in human colorectal cancer by hypericin mediated photodynamic therapy via ROS-related mechanism. Int J Biochem Cell Biol 71: 24-34, 2016.

11. Wang Q, You T, Yuan D, Han X, Hong X, He B, Wang L, Tong X, Tao L and Harris AL: Cisplatin and oxaliplatin inhibit gap junctional communication by direct action and by reduction of connexin expression, thereby counteracting cytotoxic efficacy. J Pharmacol Exp Ther 333: 903-911, 2010.

12. Yuan D, Wang Q, Wu D, Yu M, Zhang S, Li L, Tao L and Harris AL: Monocyte-endothelial adhesion is modulated by Cx43-stimulated ATP release from monocytes. Biochem Biophys Res Commun 420: 536-541, 2012.

13. Luo C, Yuan D, Zhao W, Chen H, Luo G, Su G and Hei Z: Sevoflurane ameliorates intestinal ischemia-reperfusion-induced lung injury by inhibiting the synergistic action between mast cell activation and oxidative stress. Mol Med Rep 12: 1082-1090, 2015.

14. Lee W, Belkhiri A, Lockhart AC, Merchant N, Glaeser H, Harris EI, Washington MK, Brunt EM, Zaika A, Kim RB and El-Rifai W: Overexpression of OATP1B3 confers apoptotic resistance in colon cancer. Cancer Res 68: 10315-10323, 2008.

15. Leone F, Artale S, Marino D, Cagnazzo C, Cascinu S, Pinto C, Fornarini G, Tampellini M, Di Fabio F, Sartore-Bianchi A, et al: Panitumumab in combination with infusional oxaliplatin and oral capecitabine for conversion therapy in patients with colon cancer and advanced liver metastases. The MetaPan study. Cancer 119: 3429-3435, 2013

16. Taieb J, Zaanan A, Le Malicot K, Julié C, Blons H, Mineur L, Bennouna J, Tabernero J, Mini E, Folprecht G, et al: Prognostic effect of BRAF and KRAS mutations in patients with stage III colon cancer treated with leucovorin, fluorouracil, and oxaliplatin with or without cetuximab: A post hoc analysis of the PETACC- 8 trial. JAMA Oncol: 1-11, 2016

17. Anderson BJ and Peterson LL: Systemic capillary leak syndrome in a patient receiving adjuvant oxaliplatin for locally advanced colon cancer. J Oncol Pharm Pract 22: 725-728, 2016.
18. Segretain D, Decrouy X, Dompierre J, Escalier D, Rahman N, Fiorini C, Mograbi B, Siffroi JP, Huhtaniemi I, Fenichel P and Pointis G: Sequestration of connexin43 in the early endosomes: An early event of Leydig cell tumor progression. Mol Carcinog 38: 179-187, 2003.

19. Leithe E, Sirnes S, Omori Y and Rivedal E: Downregulation of gap junctions in cancer cells. Crit Rev Oncog 12: 225-256, 2006.

20. Mesnil M, Crespin S, Avanzo JL and Zaidan-Dagli ML: Defective gap junctional intercellular communication in the carcinogenic process. Biochim Biophys Acta 1719: 125-145, 2005.

21. Kardami E, Dang X, Iacobas DA, Nickel BE, Jeyaraman M, Srisakuldee W, Makazan J, Tanguy S and Spray DC: The role of connexins in controlling cell growth and gene expression. Prog Biophys Mol Biol 94: 245-264, 2007.

22. Shao Q, Wang H, McLachlan E, Veitch GI and Laird DW: Down-regulation of $\mathrm{Cx} 43$ by retroviral delivery of small interfering RNA promotes an aggressive breast cancer cell phenotype. Cancer Res 65: 2705-2711, 2005.

23. Avanzo JL, Mesnil M, Hernandez-Blazquez FJ, Mackowiak II, Mori CM, da Silva TC, Oloris SC, Gárate AP, Massironi SM, Yamasaki $\mathrm{H}$ and Dagli ML: Increased susceptibility to urethane-induced lung tumors in mice with decreased expression of connexin43. Carcinogenesis 25: 1973-1982, 2004.

24. He B, Tong X, Wang L, Wang Q, Ye H, Liu B, Hong X, Tao L and Harris AL: Tramadol and flurbiprofen depress the cytotoxicity of cisplatin via their effects on gap junctions. Clin Cancer Res 15: 5803-5810, 2009.

25. Hong X, Wang Q, Yang Y, Zheng S, Tong X, Zhang S, Tao L and Harris AL: Gap junctions propagate opposite effects in normal and tumor testicular cells in response to cisplatin. Cancer Lett 317: 165-171, 2012.

26. Zhao Y, Liu B, Wang Q, Yuan D, Yang Y, Hong X, Wang X and Tao L: Propofol depresses the cytotoxicity of X-ray irradiation through inhibition of gap junctions. Anesth Analg 112: 1088-1095, 2011

27. Sanson M, Marcaud V, Robin E, Valéry C, Sturtz F and Zalc B: Connexin 43-mediated bystander effect in two rat glioma cell models. Cancer Gene Ther 9: 149-155, 2002. 